(n)

trobertivier

Journal of Nonlinear Mathematical Physics

ISSN (Online): 1776-0852 ISSN (Print): 1402-9251

$P^{\text {rrumisis }}$ Journal Home Page: https://www.atlantis-press.com/journals/jnmp

\title{
Integration of some examples of geodesic flows via solvable
} structures

Diego Catalano Ferraioli, Paola Morando

To cite this article: Diego Catalano Ferraioli, Paola Morando (2014) Integration of some examples of geodesic flows via solvable structures, Journal of Nonlinear Mathematical Physics 21:4, 521-532, DOI: https://doi.org/10.1080/14029251.2014.975525

To link to this article: https://doi.org/10.1080/14029251.2014.975525

Published online: 04 January 2021 


\title{
Integration of some examples of geodesic flows via solvable structures
}

\author{
Diego Catalano Ferraioli \\ Department of Mathematics, UFBA, Av Ademar de Barros $s / n$ \\ Salvador, BA CEP 40170-110, Brazil \\ diego.catalano@ufba.br \\ Paola Morando \\ DISAA, Università degli Studi di Milano, Via Celoria, 2 \\ Milano, 20133, Italy \\ paola.morando@unimi.it
}

Received 17 March 2014

Accepted 16 June 2014

\begin{abstract}
Solvable structures are particularly useful in the integration by quadratures of ordinary differential equations. Nevertheless, for a given equation, it is not always possible to compute a solvable structure. In practice, the simplest solvable structures are those adapted to an already known system of symmetries. In this paper we propose a method of integration which uses solvable structures suitably adapted to both symmetries and first integrals. In the variational case, due to Noether theorem, this method is particularly effective as illustrated by some examples of integration of the geodesic flows.
\end{abstract}

Keywords: Solvable Structures; Variational Symmetries; Euler-Lagrange Equations.

2000 Mathematics Subject Classification: 34C14, 70S10

\section{Introduction}

In recent years a great interest has been devoted to symmetry reduction methods for both ordinary and partial differential equations. This led to the development of new techniques which have given a significant improvement to the subject. It is well known that if an ordinary differential equation (ODE) $\mathscr{E}$ admits a Lie algebra of symmetries, this can be used to reduce the order of $\mathscr{E}$. This procedure, usually called Lie reduction method, is particularly useful when the symmetry algebra $\operatorname{Sym}(\mathscr{E})$ of a scalar $k^{\text {th }}$ order equation $\mathscr{E}$ admits a (non-trivial) solvable $k$-dimensional sub-algebra $\mathscr{S}$. Indeed, in view of Bianchi-Lie theorem for completely integrable distributions $[9,17,20]$, in such a case $\mathscr{E}$ can be completely integrated by quadratures. This means that one can explicitly determine, at least locally, a complete set of first integrals through the integration of a system of closed 1-forms. Unfortunately, it is not possible, in general, to compute the entire symmetry algebra $\operatorname{Sym}(\mathscr{E})$ and equations solvable by quadratures often lack local symmetries (see, e.g., [5-7, 15]). This fact raised the question of whether a generalisation of the notion of symmetry would lead to a more effective reduction method. For this reason, in recent decades, the Lie reduction method for ODEs has been extended by new techniques, which involve new kinds of symmetries such as $\lambda$ symmetries, non-local symmetries and new tools like solvable structures (see, e.g., $[1,2,5,6,11-13$, 
$15,16,18,19])$. In particular, solvable structures allowed a generalization of Bianchi-Lie theorem, with effective applications to the integration by quadratures of ODEs (see e.g. $[7,8]$ ).

A particularly important case of symmetry reduction method is provided by second order variational ODEs, with $n$-dependent variables. The reason is that variational ODEs often admit a special kind of symmetries, called variational symmetries, which are deeply connected with the existence of first integrals. Indeed, Noether theorem associates with any variational symmetry $Y$ a differentiable function $F$ which is a joint first integral for both $Y$ and the given variational equations. It follows that, on each level manifold $\Upsilon_{c}:=\{F=c\}$, the restricted variational equations are still invariant under the restriction of $Y$ to $\Upsilon_{c}$. Hence, the knowledge of a variational symmetry allows one to reduce by two the dimension of the given variational equations, whereas a purely dynamical symmetry would only lead to a one-step reduction. However, in the general case of an $h$-dimensional algebra of (non-trivial) variational symmetries $\mathscr{G}=\left\langle Y_{i}: i=1, \ldots, h\right\rangle$, one can only reduce by $h$ the dimension of the equations. In fact, the first integral $F_{i}$ associated with the generator $Y_{i}$ may not be a first integral also for the other generators of the algebra. Only when $\mathscr{G}$ is an $n$-dimensional Abelian algebra of Noether symmetries it is possible to ensure that $Y_{i}\left(F_{j}\right)=0, \forall i, j \in\{1, . ., n\}$, and then use Bianchi-Lie theorem on each $\Upsilon_{c}$ to completely integrate by quadratures the given equations. However, in some cases it may be difficult to compute all necessary symmetries to apply the Bianchi-Lie or Liouville theorems.

In this paper we propose an alternative integration method for variational ODEs based on the computation of solvable structures which are suitably adapted to some variational symmetries and tangent to the level manifolds of some associated first integrals. Once such a solvable structure is found, we can get the solution to the variational ODEs by quadratures. It is worth remarking that under the hypothesis of Lie-Bianchi or Liouville theorems, our method may lead to the same integration procedure given by these theorems. However, the proposed method can be applied to more general situations exploiting the knowledge of non-Abelian algebras of variational symmetries. In such cases, the freedom one has in the search of a solvable structure adapted to certain variational symmetries and level manifolds of first integrals, makes computations more feasible and may lead to a more general integration procedure. Although the proposed method is not algorithmic, the analysis of the differential equations which determine the solvable structures for a given system of ODE is quite feasible, especially by using packages for symbolic manipulation.

The paper is organized as follows. In Section 2, for the convenience of the reader, we collect some notations and basic facts on the geometry of differential equations and solvable structures. In Section 3 we give a description of the proposed method together with explicit applications to some examples of variational ODEs.

\section{Preliminaries}

Throughout the paper we assume that the reader is familiar with the geometry of differential equations. Further details can be found in $[4,10,17,20]$. We routinely use Einstein convention and understand summation over repeated indices unless otherwise specified.

\subsection{ODEs as sub-manifolds of jet spaces}

Let $M$ be an $n$-dimensional manifold, with local coordinates $\left\{x_{i}\right\}$. Given a trivial fiber bundle $\pi$ : $\mathbb{R} \times M \rightarrow \mathbb{R}$, we denote by $\pi_{k}: J^{k}(\pi) \rightarrow M$ the $k^{\text {th }}$ order jet bundle associated with $\pi$, and by $j_{k}(s)$ 
the $k^{\text {th }}$ order jet prolongation of a section $s$ of $\pi$. The $k^{\text {th }}$ order jet space $J^{k}(\pi)$ is a $(1+n+k n)-$ dimensional manifold which is naturally equipped with the contact (or Cartan) distribution $\mathscr{C}^{k}$ of tangent planes to graphs of $k^{\text {th }}$ order jet prolongations $j_{k}(s)$. In this framework, a $k^{\text {th }}$ order system of ordinary differential equations can be regarded as a sub-manifold $\mathscr{E} \subset J^{k}(\pi)$ and its solutions are sections of $\pi$ whose $k^{\text {th }}$ order prolongations are integral manifolds of $\left.\mathscr{C}^{k}\right|_{\mathscr{E}}$, the restriction to $\mathscr{E}$ of $\mathscr{C}^{k}$.

In this paper we only deal with systems of second order Euler-Lagrange equations, which are associated with a first order Lagrangian $L \in C^{\infty}\left(J^{1}(\pi)\right)$. We suppose that $L$ is a regular Lagrangian, i.e., the corresponding Legendre transformation is non degenerate. We denote by $\left\{t, x_{i}, v_{i}\right\}$ the canonical local coordinates on $J^{1}(\pi)$ and we assume that $\mathscr{E}$ is a determined second order system in normal form. In this case, the solutions to $\mathscr{E}$ are integral lines of the following vector field on $\mathscr{E}$

$$
\bar{D}_{t}=\frac{\partial}{\partial t}+v_{i} \frac{\partial}{\partial x_{i}}+\ldots+f_{i}\left(t, x_{j}, v_{j}\right) \frac{\partial}{\partial v_{i}},
$$

where $f_{i}\left(t, x_{j}, v_{j}\right)$ are exactly the right-hand sides of the equations defining $\mathscr{E}$ in normal form. A function $F \in C^{\infty}(\mathscr{E})$ is said to be a first integral of $\mathscr{E}$ iff $\bar{D}_{t}(F)=0$. The Euler-Lagrange equations for a first order Lagrangian $L$ can be written in the following form:

$$
D_{t}\left(\frac{\partial L}{\partial v_{i}}\right)-\frac{\partial L}{\partial x_{i}}=0, \quad i=1, \ldots, n,
$$

where $D_{t}$ is the total derivative operator. If $L$ is regular, (2.2) is a determined system which can be put in normal form.

\subsection{Symmetries and variational symmetries}

A vector field $Y$ on $J^{k}(\pi)$ is called a Lie symmetry if $Y$ is an infinitesimal symmetry of $\mathscr{C}^{k}$. Lie symmetries can be divided in two classes (see [17,20]): Lie point symmetries and contact symmetries, obtained by prolonging vector fields $X$ on $J^{0}(\pi):=\mathbb{R} \times M$ and $J^{1}(\pi)$, respectively. In particular, the first prolongation of a vector field $X=\xi \partial_{t}+\eta_{i} \partial_{x_{i}}$ on $J^{0}(\pi)$, is the vector field $X^{(1)}=X+\left(D_{t} \eta_{i}-v_{i} D_{t} \xi\right) \partial_{v_{i}}$ on $J^{1}(\pi)$.

Given a $k^{\text {th }}$ order ODEs $\mathscr{E}$, the symmetries of $\mathscr{C}^{k}$ which are tangent to $\mathscr{E}$ are called (external) classical symmetries of $\mathscr{E}$. Hence, by restricting to $\mathscr{E}$ a classical symmetry $Y$ of $\mathscr{E}$, one gets a vector field $\bar{Y}$ such that $\left[\bar{Y}, \bar{D}_{t}\right]=h \bar{D}_{t}$, where $h$ is a suitable function on $\mathscr{E}$. More in general, a vector field $Z$ on $\mathscr{E}$ satisfying $\left[Z, \bar{D}_{t}\right]=h \bar{D}_{t}$ is called a dynamical symmetry. Notice that in general there may exist dynamical symmetries which cannot be obtained by restricting to $\mathscr{E}$ a symmetry of $\mathscr{C}^{k}$.

Given a regular first order Lagrangian $L \in C^{\infty}\left(J^{1}(\pi)\right)$, the corresponding Poincaré-Cartan form is the 1 -form locally written as

$$
\Theta:=\frac{\partial L}{\partial v_{i}}\left(d x_{i}-v_{i} d t\right)+L d t, \quad i=1, \ldots, n
$$

(see [14] for an intrinsic definition of $\Theta$ and its general properties). If $L$ is regular, it is easy to show that the 2 -form $d \Theta$ has a 1-dimensional annihilator generated by the vector field $\bar{D}_{t}$.

Definition 2.1. Let $\Theta$ be the Poincaré-Cartan form (2.3) for a first order regular Lagrangian $L \in$ $C^{\infty}\left(J^{1}(\pi)\right)$. A vector field $Y$ on $J^{1}(\pi)$ is a variational Cartan symmetry for $L$ iff $\mathscr{L}_{Y} \Theta=d f$, for some $f \in C^{\infty}\left(J^{1}(\pi)\right)$. When $\mathscr{L}_{Y} \Theta=0$ and $Y$ is a point symmetry, then $Y$ is called a Noether symmetry. In 
the following, for ease of discussion, we will call variational symmetries both Cartan and Noether symmetries.

Any variational symmetry $Y$ for a regular Lagrangian $L$ is also a symmetry for the corresponding Euler-Lagrange equations. Moreover Noether theorem associates with any variational symmetry $Y$ the first integral $F=f-Y\lrcorner \Theta$ of the Euler-Lagrange equations.

\subsection{Distributions and solvable structures}

Given a set of vector fields $\left\{Y_{1}, \ldots, Y_{n-k}\right\}$ on a $n$-dimensional manifold $N$ we denote by $\mathscr{D}:=$ $\left\langle Y_{1}, \ldots, Y_{n-k}\right\rangle$ the distribution of vector fields generated by $Y_{1}, \ldots, Y_{n-k}$. The distribution $\mathscr{D}$ is completely integrable (in Frobenious sense) iff $[X, Y] \in \mathscr{D}, \forall X, Y \in \mathscr{D}$. Let $U$ be an open domain of $N$ where the vector fields $\left\{Y_{1}, \ldots, Y_{n-k}\right\}$ are pointwise linearly independent. Then $\mathscr{D}$ is a $(n-k)$-dimensional distribution on $U$ and, if $\mathscr{D}$ is completely integrable, $\mathscr{D}$ determines a $(n-k)$ dimensional foliation of $U \subseteq N$. Given a distribution $\mathscr{D}$, a vector field $X$ is a symmetry of $\mathscr{D}$ iff $[X, Y] \in \mathscr{D}$, for all $Y \in \mathscr{D}$. Let $\mathscr{D}$ and $\mathscr{Y}$ be two distributions on $N$. We say that $\mathscr{D}$ and $\mathscr{Y}$ are transversal at $p \in N$ iff they do not vanish at $p$ and $\mathscr{D}(p) \cap \mathscr{Y}(p)=\{0\}$. Analogously, $\mathscr{D}$ and $\mathscr{Y}$ are called transversal iff they are transversal at any point. An algebra of symmetries for a distribution $\mathscr{D}$ will be called nontrivial if it generates a distribution which is transversal to $\mathscr{D}$.

It is well known that, given a 1-dimensional distribution $\mathscr{D}$ on an $n$-dimensional manifold $N$, the knowledge of a solvable $(n-1)$-dimensional algebra $\mathscr{G}$ of nontrivial symmetries for $\mathscr{D}$ guarantees that $\mathscr{D}$ can be integrated by quadratures. The notion of solvable structure provides a generalization of this classical integrability result $([1,2,13])$.

Definition 2.2. Let $\mathscr{D}$ be a 1-dimensional distribution on an $n$-dimensional manifold $N$. A set of vector fields $\mathscr{Z}:=\left\{Z_{1}, Z_{2}, \ldots, Z_{r}\right\}$, with $r \leq n-1$, is a solvable structure for $\mathscr{D}$ in an open neighborhood $U \subseteq N$ iff, by denoting $\mathscr{D}_{0}:=\mathscr{D}$ and $\mathscr{D}_{h}:=\mathscr{D} \oplus\left\langle Z_{1}, \ldots, Z_{h}\right\rangle$, with $h \leq r$, the following conditions are satisfied:

(1) $\left\langle Z_{1}, Z_{2}, \ldots, Z_{h}\right\rangle$ is $h$-dimensional and transversal to $\mathscr{D}$ in $U$, for any $h \leq r$;

(2) $\mathscr{D}_{r}$ is an $(r+1)$-dimensional distribution on $U$;

(3) $\mathscr{L}_{Z_{h}} \mathscr{D}_{h-1} \subset \mathscr{D}_{h-1}, \forall h \in\{1, \ldots, r\}$.

A solvable structure for $\mathscr{D}$ is called maximal iff $r=n-1$, in which case $\mathscr{D}_{n-1}=T U$.

Remark 2.1. It is clear from Definition 2.2 , that in principle a maximal solvable structure for $\mathscr{D}$ always exists, in a neighborhood of every non-singular point for $\mathscr{D}$. Nevertheless, for a given distribution $\mathscr{D}$, it may be difficult to find such a structure explicitly.

\section{Adapted Solvable Structures for the Integration of Variational ODEs}

Let $M$ be a smooth manifold of dimension $(2 n+1)$, equipped with a 1-dimensional distribution $\mathscr{D}=\langle X\rangle$. For any $h$-tuple $\mathbf{F}:=\left(F_{1}, \ldots, F_{h}\right) \in C^{\infty}(M)^{h}$ of smooth functions, let

$$
U_{\mathbf{F}}:=\left\{p \in M \mid \bigwedge_{i=1}^{h} d_{p} F_{i} \neq 0\right\} \subseteq M
$$

and consider the $(2 n-h+1)$-dimensional foliation of $U_{\mathbf{F}}$ whose leaves are the level sets

$$
\Upsilon_{c}:=\left\{p \in U_{\mathbf{F}} \mid \mathbf{F}(p)=\mathbf{c}\right\},
$$


parameterized by $\mathbf{c} \in \mathbb{R}^{h}$.

Lemma 3.1. Let $\mathscr{Z}:=\left\{Z_{1}, \ldots, Z_{2 n-h}\right\}$ be a solvable structure for $\mathscr{D}$ in $M$. If $\mathbf{F}$ is made of first integrals of $\mathscr{D}$ and $Z_{a}\left(F_{i}\right)=0 \forall a=1, \ldots, 2 n-h$ and $i=1, \ldots, h$, then $\mathscr{Z}_{\mathbf{c}}:=\left\{Z_{1}\left|\mathrm{r}_{c}, \ldots, Z_{2 n-h}\right| \mathrm{r}_{c}\right\}$ is a maximal solvable structure for $\mathscr{D}_{\mathbf{c}}:=\left\langle X \mid \Upsilon_{c}\right\rangle$ in $\Upsilon_{c}$ for all $\mathbf{c} \in \mathbb{R}^{h}$.

Proof. The proof is an immediate consequence of the fact that $\Upsilon_{c}$ is a $(2 n-h+1)$-dimensional manifold and $\left.Z\right|_{\Upsilon_{c}}$ is tangent to $\Upsilon_{c}$.

Definition 3.1. A solvable structure $\mathscr{Z}=\left\{Z_{1}, \ldots, Z_{2 n-h}\right\}$ fulfilling hypothesis of Lemma 3.1 is called adapted to $\mathbf{F}$.

Theorem 3.1. Let $\Omega \in \Lambda^{2 n-h+1}(M)$ be a form such that $\Omega_{\mathbf{c}}:=\left.\Omega\right|_{r_{c}}$ is a volume form on $\Upsilon_{c}$, for all $\mathbf{c} \in \mathbb{R}^{h}$, and let $\mathscr{Z}$ be a solvable structure for $\mathscr{D}=\langle X\rangle$ adapted to $\mathbf{F}$. Then the 1 -forms

$$
\Omega_{i}:=\frac{\left.\left.\left.\left.\left.Z_{1}\right\lrcorner \ldots \hat{Z}_{i}\right\lrcorner \ldots\right\lrcorner Z_{2 n-h}\right\lrcorner X\right\lrcorner \Omega}{\left.\left.\left.\left.Z_{1}\right\lrcorner \ldots\right\lrcorner Z_{2 n-h}\right\lrcorner X\right\lrcorner \Omega}, i=1,2, \ldots 2 n-h
$$

fulfill the following properties:

- $\Omega_{2 n-h} \mid \mathrm{r}_{c}=d I_{2 n-h}^{c}$, for some $I_{2 n-h}^{c} \in C^{\infty}\left(\Upsilon_{c}\right)$;

- $\left.\Omega_{2 n-h-1}\right|_{c} \cap\left\{I_{2 n-h}^{c}=c_{2 n-h}\right\}=d I_{2 n-h-1}^{c}$, for some $I_{2 n-h-1}^{c} \in C^{\infty}\left(\Upsilon_{c} \cap\left\{I_{2 n-h}^{c}=c_{2 n-h}\right\}\right)$;

- ...

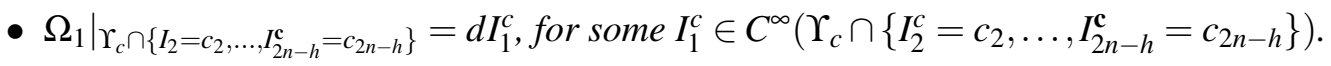

Proof. Being $\mathscr{Z}$ a solvable structure adapted to $\mathbf{F}, \mathscr{Z}_{\mathbf{c}}$ is a maximal solvable structure for $\mathscr{D}_{\mathbf{c}}$ on $\Upsilon_{c}$ for all $\mathbf{c} \in \mathbb{R}^{h}$ (see Lemma 3.1 above). Therefore we can apply the results of $[1,2,8]$ to $\mathscr{Z}_{\mathbf{c}}$ and the conclusion follows immediately.

Corollary 3.1. Let $\mathscr{Z}$ be a solvable structure adapted to $\mathbf{F}$. Then, for any $\mathbf{c} \in \mathbb{R}^{h}$, one can explicitly compute $2 n-h$ first integrals for the 1 -dimensional distribution $\mathscr{D}_{\mathbf{c}}$ on the $(2 n+h-1)$-dimensional level set $\Upsilon_{c}$.

Proof. The proof is an immediate consequence of Theorem 3.1.

In the following we apply Theorem 3.1 to the special case of variational ODEs for a regular Lagrangian $L$, exploiting both variational symmetries $Y_{i}$ and their associated first integrals $\mathbf{F}=\left\{F_{i}\right\}$. The method is not algorithmic, but the freedom we have in the choice of the first integrals $\mathbf{F}$ may help in finding an adapted solvable structure in a suitable open neighborhood of $U_{\mathbf{F}}$. In fact, in general, the first integral $F_{i}$ associated with the generator $Y_{i}$ is not a first integral for all the other generators of the algebra and this is the moment when the method begins to branch into several possibilities. If we opt for small dimensional leaves $\Upsilon_{c}$ (by using a large number of first integrals $\left.F_{i}\right)$, then we have to find a small dimensional solvable structure, but we also have fewer symmetries still available to be included in it. On the other hand, if we choose $\Upsilon_{c}$ of higher dimension, we may have a bigger number of residual symmetries to be used in the construction of the adapted solvable structure, but the number of the vector fields to be found in order to complete the solvable structure increases as well.

It is worth remarking that, even if there is no evidence that this integration method is more effective than the other ones, the analysis of the differential equations which determine the solvable 
structures for a system of ODE is often feasible, in particular by using a package for symbolic manipulation like those written for Maple. Among the several useful packages for the geometric study of differential equations, we suggest the packages "DifferentialGeometry" and "Jets" by I. Anderson (and coworkers) and M. Marvan, respectively. The first package, which is included in the current versions of Maple, is recommended for various computations involved in the determination of solvable structures. The second package, which can be downloaded from the official web page http://jets.math.slu.cz, is very helpful, for example, in the computation of symmetries.

The following three examples provide an explicit application of the method of adapted solvable structures in the integration of variational ODEs. In particular, Example 1 shows that, when a $n$-dimensional Abelian algebra of variational symmetries for a system of $n$ Euler-Lagrange equations is known, our procedure reduces to the Liouville theorem. On the other hand, Example 2 and Example 3 provide an application of the proposed method to the integration of systems of $n$ variational ODEs by using a solvable structure which is not an $n$-dimensional Abelian symmetry algebra. Moreover, since the first two examples deal with purely kinetic Lagrangians $L=\frac{1}{2} g_{i j} v_{i} v_{j}$ (no summation over repeated indices), with $g=g_{i j} d x_{i} d x_{j}$ being one of the Riemannian metrics classified by Bianchi in [3], these examples also provide applications of our method to the integration by quadratures of geodesic flows.

In all the examples we consider $M=\mathbb{R}^{3}$ and hence $J^{1}(\pi)$ is the 7-dimensional manifold with local coordinates $\left(t, x_{1}, x_{2}, x_{3}, v_{1}, v_{2}, v_{3}\right)$. We consider the distribution $\mathscr{D}=\left\langle\bar{D}_{t}\right\rangle$, where $\bar{D}_{t}$ is given by (2.1) so that the solutions to the Euler-Lagrange equations correspond to the trajectories of $\bar{D}_{t}$. We note that the proposed method is mainly of local nature as, in general, we may be able to find an adapted solvable structure for $\mathscr{D}$ only on some open neighborhood of $U_{\mathbf{F}}$.

Example 1. Consider the regular Lagrangian

$$
L=\frac{1}{2} v_{1}^{2}+\frac{1}{2} v_{2}^{2}+x_{1} v_{2} v_{3}+\frac{1+x_{1}^{2}}{2} v_{3}^{2},
$$

describing the geodesic flow of the Riemannian metric $g=d x_{1}^{2}+d x_{2}^{2}+2 x_{1} d x_{2} d x_{3}+\left(1+x_{1}^{2}\right) d x_{3}^{2}$ (see Category E metric, in the English translation of [3], which is of Bianchi type II with a transitive group $G_{4}$ ). The Lagrangian admits the Abelian symmetries algebra generated by the vector fields $Y_{0}:=\partial_{t}, Y_{1}:=\partial_{x_{2}}, Y_{2}:=\partial_{x_{3}}$, with associated first integrals $F_{0}:=L, F_{1}:=-v_{2}-x_{1} v_{3}, F_{2}:=-x_{1} v_{2}-$ $\left(1+x_{1}^{2}\right) v_{3}$.

In this case

$$
\begin{aligned}
\bar{D}_{t}= & \partial_{t}+v_{1} \partial_{x_{1}}+v_{2} \partial_{x_{2}}+v_{3} \partial_{x_{3}}+\left(v_{2} v_{3}+x_{1} v_{3}^{2}\right) \partial_{v_{1}}+ \\
& \left(-v_{3} v_{1}+x_{1} v_{1} v_{2}+x_{1}^{2} v_{1} v_{3}\right) \partial_{v_{2}}-\left(v_{1} v_{2}+x_{1} v_{1} v_{3}\right) \partial_{v_{3}} .
\end{aligned}
$$

We consider the 4-dimensional level manifolds $\Upsilon_{c}:=\left\{F_{0}=c_{0}, F_{1}=c_{1}, F_{2}=c_{2}\right\}$ which can be equivalently presented as

$$
\Upsilon_{c}=\left\{\begin{array}{l}
v_{1}=\varepsilon_{1} \sqrt{-c_{1}^{2} x_{1}^{2}+2 c_{1} c_{2} x_{1}-c_{1}^{2}-c_{2}^{2}+2 c_{0}}, \quad \varepsilon_{1}= \pm 1, \\
v_{2}=-c_{1} x_{1}^{2}+c_{2} x_{1}-c_{1}, \\
v_{3}=c_{1} x_{1}-c_{2},
\end{array}\right.
$$

so that $\left\{t, x_{1}, x_{2}, x_{3}\right\}$ can be taken as internal coordinates on $\Upsilon_{c}$, and $\Omega_{c}=d t \wedge d x_{1} \wedge d x_{2} \wedge d x_{3}$ as the corresponding volume form. 
Obviously, the vector fields $\bar{D}_{t}, Y_{0}, Y_{1}$ and $Y_{2}$ are tangent to $\Upsilon_{c}$ and $\left\{Y_{0}, Y_{1}, Y_{2}\right\}$ form a maximal Abelian solvable structure for $\mathscr{D}$ on $\Upsilon_{c}$, whenever

$$
\left.\left.\left.\left.\Delta:=Y_{0}\right\lrcorner Y_{1}\right\lrcorner Y_{2}\right\lrcorner \bar{D}_{t}\right\lrcorner \Omega_{c}=\varepsilon_{1} \sqrt{-c_{1}^{2} x_{1}^{2}+2 c_{1} c_{2} x_{1}-c_{1}^{2}-c_{2}^{2}+2 c_{0}} \neq 0 .
$$

Therefore Corollary 3.1 allows us to integrate the Euler-Lagrange equations corresponding to the Lagrangian (3.4), on $U_{\mathbf{F}}:=\{\Delta \neq 0\}$. Indeed, Theorem 3.1, with $n=h=3$, guarantees that on $U_{\mathbf{F}}$ the restricted 1-forms $\Omega_{i} \mid \Upsilon_{c}(i=0,1,2)$ are closed and can be written as $\Omega_{i} \mid \Upsilon_{c}=d I_{i}$, with

$$
\begin{aligned}
& I_{0}= \varepsilon_{1} \arcsin \left(\frac{c_{2}-c_{1} x_{1}}{\sqrt{2 c_{0}-c_{1}^{2}}}\right)-t, \\
& I_{1}= \frac{\varepsilon_{1}\left(x_{1} c_{1}+c_{2}\right) \sqrt{c_{1}^{2} x_{1}^{2}+2 c_{2} c_{1} x_{1}-c_{1}^{2}-c_{2}^{2}+2 c_{0}}}{2 c_{1}^{2}} \\
&-\frac{\varepsilon_{2}\left(2 c_{0}+c_{1}^{2}\right) \arctan \left(\frac{\left(\varepsilon_{2} c_{1} x_{1}-\varepsilon_{2} c_{2}\right)}{\varepsilon_{1} \sqrt{-c_{1}^{2} x_{1}^{2}+2 c_{2} c_{1} x_{1}-c_{1}^{2}-c_{2}^{2}+2 c_{0}}}\right)}{2 c_{1}^{2}}-x_{2}, \\
& I_{2}=x_{3}+\varepsilon_{1} \frac{\sqrt{-c_{1}^{2} x_{1}^{2}+2 c_{2} c_{1} x_{1}-c_{1}^{2}-c_{2}^{2}+2 c_{0}}}{c_{1}}
\end{aligned}
$$

where $\varepsilon_{2}=\operatorname{sgn}\left(c_{1}\right)= \pm 1$. Hence, on $U_{\mathbf{F}}$, the solution to the Euler-Lagrange equations is given implicitly by $\left\{F_{i}=c_{i}, I_{i}=h_{i}, \quad i=0,1,2\right\}$.

Example 2. Given a smooth non constant function $\phi\left(x_{1}\right)$, let us consider the regular Lagrangian

$$
L=\frac{1}{2} v_{1}^{2}+\frac{1}{2} \phi\left(x_{1}\right)^{2}\left(v_{2}^{2}+e^{2 x_{2}} v_{3}^{2}\right)
$$

describing the geodesic flow of the Riemannian metric $g=d x_{1}^{2}+\phi\left(x_{1}\right)^{2}\left(d x_{2}^{2}+e^{2 x_{2}} d x_{3}^{2}\right)$ (see Category $\mathrm{C}$ metrics, in the English translation of [3], which correspond to the case of an intransitive group $G_{3}$ ). In this case the full symmetry algebra is generated by the vector fields

$$
X_{0}:=\partial_{t}, \quad X_{1}:=\partial_{x_{3}}, \quad X_{2}:=\partial_{x_{2}}-x_{3} \partial_{x_{3}}, \quad X_{3}:=x_{3} \partial_{x_{2}}+\frac{1}{2}\left(e^{-2 x_{2}}-x_{3}^{2}\right) \partial_{x_{3}},
$$

with associated first integrals

$$
\begin{aligned}
& F_{0}:=L, \quad F_{1}=-\phi\left(x_{1}\right)^{2} e^{2 x_{2}} v_{3}, \\
& F_{2}:=\phi\left(x_{1}\right)^{2}\left(x_{3} e^{2 x_{2}} v_{3}-v_{2}\right), \quad F_{3}:=\frac{1}{2} \phi\left(x_{1}\right)^{2}\left(-v_{3}+e^{2 x_{2}} x_{3}^{2} v_{3}-2 x_{3} v_{2}\right) .
\end{aligned}
$$

In this case we have

$$
\begin{aligned}
\bar{D}_{t}=\partial_{t}+ & v_{1} \partial_{x_{1}}+v_{2} \partial_{x_{2}}+v_{3} \partial_{x_{3}}+\phi\left(x_{1}\right) \phi^{\prime}\left(x_{1}\right)\left(v_{2}^{2}+e^{2 x_{2}} v_{3}^{2}\right) \partial_{v_{1}} \\
& +\frac{\left(e^{2 x_{2}} v_{3}^{2} \phi\left(x_{1}\right)-2 v_{1} v_{2} \phi^{\prime}\left(x_{1}\right)\right)}{\phi\left(x_{1}\right)} \partial_{v_{2}}-\frac{2 v_{3}\left(v_{1} \phi^{\prime}\left(x_{1}\right)+v_{2} \phi\left(x_{1}\right)\right)}{\phi\left(x_{1}\right)} \partial_{v_{3}} .
\end{aligned}
$$

Contrary to the previous case, the vector fields $\left\{X_{0}, X_{1}, X_{2}, X_{3}\right\}$ are not tangent to the level manifolds of the first integrals $F_{0}, F_{1}, F_{2}, F_{3}$. This is a consequence of the fact that, in this case, the symmetry 
algebra is not Abelian, being

$$
\begin{aligned}
& {\left[X_{0}, X_{1}\right]=0, \quad\left[X_{0}, X_{2}\right]=0, \quad\left[X_{0}, X_{3}\right]=0,} \\
& {\left[X_{1}, X_{2}\right]=-X_{1},\left[X_{1}, X_{3}\right]=X_{2},\left[X_{2}, X_{3}\right]=-X_{3} .}
\end{aligned}
$$

To give an idea of the choices one is required to make when applying the proposed method, we show here two different approaches to the integration of the distribution $\mathscr{D}$.

Approach 1. If we consider the 4-dimensional level manifolds $\Upsilon_{c}:=\left\{F_{i}=c_{i}, \quad i=0,1,2\right\}$, we have to look for a solvable structure $\left\{Y_{0}, Y_{1}, Y_{2}\right\}$ for $\mathscr{D}$ adapted to $\mathbf{F}:=\left(F_{0}, F_{1}, F_{2}\right)$.

This solvable structure can be easily computed by making some simple ansatz. For example, choosing $Y_{0}:=X_{0}$, in general we have to look for a vector field $Y_{1}$ which is a symmetry of $\left\langle\bar{D}_{t}, Y_{0}\right\rangle$. To this end, we impose the conditions

$$
\left[Y_{1}, \bar{D}_{t}\right]=0, \quad Y_{1}\left(F_{3}\right)=0, \quad\left[Y_{1}, Y_{0}\right]=0
$$

on $Y_{1}$ and immediately find the Cartan symmetry

$$
Y_{1}=\phi\left(x_{1}\right)^{2}\left(v_{2} \partial_{x_{2}}+v_{3} \partial_{x_{3}}+e^{2 x_{2}} v_{3}^{2} \partial_{v_{2}}-2 v_{2} v_{3} \partial_{v_{3}}\right) .
$$

Then we have to find a symmetry $Y_{2}$ of $\left\langle\bar{D}_{t}, Y_{0}, Y_{1}\right\rangle$, but assuming that $Y_{2}$ is a symmetry of $\left\langle Y_{1}\right\rangle$, it is not difficult to find, for example,

$$
Y_{2}=-e^{2 x_{2}} v_{3} \frac{\phi\left(x_{1}\right)^{3}}{\phi^{\prime}\left(x_{1}\right)} \partial_{x_{1}}+\phi\left(x_{1}\right)^{2} e^{2 x_{2}} v_{3} \partial_{x_{2}}-\phi\left(x_{1}\right)^{2} v_{2} \partial_{x_{3}}+\phi\left(x_{1}\right)^{2} e^{2 x_{2}} v_{2} v_{3} \partial_{v_{2}} .
$$

Now, since

$$
\Upsilon_{c}=\left\{\begin{array}{l}
v_{1}=\frac{\varepsilon_{1}}{\phi\left(x_{1}\right)} \sqrt{2 c_{0} \phi\left(x_{1}\right)^{2}-\left(c_{1}^{2} e^{-2 x_{2}}+\left(c_{2}+c_{1} x_{3}\right)^{2}\right)}, \quad \varepsilon_{1}= \pm 1 \\
v_{2}=-\frac{c_{1} x_{3}+c_{2}}{\phi\left(x_{1}\right)^{2}} \\
v_{3}=-\frac{c_{1}}{\phi\left(x_{1}\right)^{2} e^{2 x_{2}}},
\end{array}\right.
$$

it is possible to choose $\left\{t, x_{1}, x_{2}, x_{3}\right\}$ as internal coordinates on $\Upsilon_{c}$, and $\Omega_{c}=d t \wedge d x_{1} \wedge d x_{2} \wedge d x_{3}$ as the corresponding volume form. Then, on $U_{\mathbf{F}}=\{\Delta \neq 0\}$, where

$$
\begin{aligned}
\Delta & \left.\left.\left.\left.=Y_{0}\right\lrcorner Y_{1}\right\lrcorner Y_{2}\right\lrcorner \bar{D}_{t}\right\lrcorner \Omega_{c} \\
& =\frac{\varepsilon_{1}}{\phi\left(x_{1}\right)}\left(c_{1}^{2} e^{-2 x_{2}}+\left(c_{2}+c_{1} x_{3}\right)^{2}\right) \sqrt{2 c_{0} \phi\left(x_{1}\right)^{2}-\left(c_{1}^{2} e^{-2 x_{2}}+\left(c_{2}+c_{1} x_{3}\right)^{2}\right)},
\end{aligned}
$$

we can use the solvable structure $\left\{Y_{0}, Y_{1}, Y_{2}\right\}$ to find three independent first integrals for the EulerLagrange equations associated with (3.6) according to the general method described in Corollary 3.1. Indeed, by Theoren 3.1, with $n=h=3$, on $U_{\mathbf{F}}$ the restricted 1-form $\Omega_{2} \mid \mathrm{r}_{c}$ is closed and can be 
written as $\Omega_{2} \mid \mathrm{r}_{c}=d I_{2}$, where

$$
I_{2}=c_{1}^{2} e^{-2 x_{2}}+\left(c_{2}+c_{1} x_{3}\right)^{2}
$$

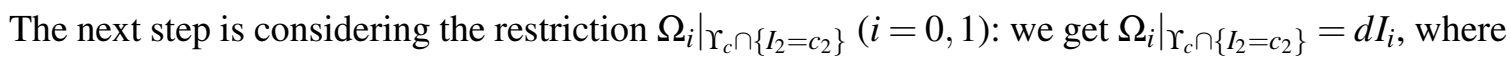

$$
\begin{aligned}
& I_{0}=t-\varepsilon_{1} \int \frac{\phi\left(x_{1}\right)}{\sqrt{2 c_{0} \phi\left(x_{1}\right)^{2}-h_{2}}} d x_{1}, \\
& I_{1}=-\frac{\operatorname{arctanh}\left(\frac{c_{1} x_{3}+c_{2}}{\sqrt{h_{2}}}\right)}{\sqrt{h_{2}}}-\varepsilon_{1} \int \frac{1}{\phi\left(x_{1}\right) \sqrt{2 c_{0} \phi\left(x_{1}\right)^{2}-h_{2}}} d x_{1} .
\end{aligned}
$$

Hence, on $U_{\mathbf{F}}$, the solutions to the Euler-Lagrange equations can be implicitly described as $\left\{F_{i}=c_{i}, I_{i}=h_{i}, i=0,1,2\right\}$.

Approach 2. Here we apply Corollary 3.1 with $n=3$ and $h=4$. More precisely, we look for a solvable structure adapted to $\mathbf{F}:=\left(F_{0}, F_{1}, F_{2}, F_{3}\right)$ i.e., which is tangent to the 3-dimensional level manifolds $\bar{\Upsilon}_{c}:=\left\{F_{i}=c_{i}, i=0,1,2,3\right\}$. Obviously the vector fields $\bar{D}_{t}$ and $Y_{0}$ are tangent to $\bar{\Upsilon}_{c}$, and we have to find a single vector field $Y_{1}$ which is tangent to $\bar{\Upsilon}_{c}$ and is also a symmetry for $\left\langle\bar{D}_{t}, Y_{0}\right\rangle$. An example of such a vector field is the Cartan symmetry $Y_{1}$ found in Approach 1.

Now, since

$$
\bar{\Upsilon}_{c}=\left\{\begin{array}{l}
v_{1}=\frac{\varepsilon_{1} \sqrt{2 c_{0} \phi\left(x_{1}\right)^{2}-c_{2}^{2}-2 c_{1} c_{3}}}{\phi\left(x_{1}\right)}, \quad \varepsilon_{1}= \pm 1 \\
v_{2}=-\frac{c_{1} x_{3}+c_{2}}{\phi\left(x_{1}\right)^{2}}, \\
v_{3}=\frac{c_{1} x_{3}^{2}+2 x_{3} c_{2}-2 c_{3}}{\phi\left(x_{1}\right)^{2}}, \\
x_{2}=\frac{1}{2} \ln \left(-\frac{c_{1}}{c_{1} x_{3}^{2}+2 c_{2} x_{3}-2 c_{3}}\right),
\end{array}\right.
$$

we can choose $\left\{t, x_{1}, x_{3}\right\}$ as internal coordinates in $\bar{\Upsilon}_{c}$ and $\Omega_{c}=d t \wedge d x_{1} \wedge d x_{3}$ as the corresponding volume form. Hence, $\left\{Y_{0}, Y_{1}\right\}$ is a solvable structure for $\mathscr{D}$ on $\bar{\Upsilon}_{c}$, whenever

$$
\left.\left.\left.\Delta=Y_{0}\right\lrcorner Y_{1}\right\lrcorner \bar{D}_{t}\right\lrcorner \Omega_{c}=\frac{\varepsilon_{1}\left(c_{1} x_{3}^{2}+2 x_{3} c_{2}-2 c_{3}\right) \sqrt{2 c_{0} \phi\left(x_{1}\right)^{2}-c_{2}^{2}-2 c_{1} c_{3}}}{\phi\left(x_{1}\right)} \neq 0 .
$$

Moreover, in $U_{\mathbf{F}}=\{\Delta \neq 0\}$, the restricted 1-forms $\left.\Omega_{i}\right|_{\bar{\Upsilon}_{c}}(i=0,1)$ are closed and can be written as $\left.\Omega_{i}\right|_{\bar{\Upsilon}_{c}}=d I_{i}$, where

$$
\begin{aligned}
& I_{0}=t-\varepsilon_{1} \int \frac{\phi\left(x_{1}\right)}{\sqrt{2 c_{0} \phi\left(x_{1}\right)^{2}-c_{2}^{2}-2 c_{1} c_{3}}} d x_{1}, \\
& I_{1}=-\frac{\operatorname{arctanh}\left(\frac{c_{1} x_{3}+c_{2}}{\sqrt{c_{2}^{2}+2 c_{1} c_{3}}}\right)}{\sqrt{c_{2}^{2}+2 c_{1} c_{3}}}-\varepsilon_{1} \int \frac{1}{\phi\left(x_{1}\right) \sqrt{2 c_{0} \phi\left(x_{1}\right)^{2}-c_{2}^{2}-2 c_{1} c_{3}}} d x_{1},
\end{aligned}
$$


in view of Theorem 3.1, with $n=3$ and $h=4$. This means that, on the considered domains, the solutions to the Euler-Lagrange equations can be implicitly described as $\left\{F_{0}=c_{0}, F_{1}=c_{1}, F_{2}=c_{2}, F_{3}=c_{3}, I_{0}=h_{0}, I_{1}=h_{1}\right\}$.

Remark 3.1. Despite the fact that $Y_{1}$ is a Cartan symmetry, the associated first integral cannot be used for the complete integration of the geodesic flow. In fact $L_{Y_{1}}(\Theta)=d\left(\frac{1}{2} \phi^{2}\left(v_{2}^{2}+e^{2 x_{2}} v_{3}^{2}\right)\right)$, and the first integral associated with $Y_{1}$ is

$$
\left.F_{4}=\frac{1}{2} \phi^{2}\left(v_{2}^{2}+e^{2 x_{2}} v_{3}^{2}\right)-Y_{1}\right\lrcorner \Theta=-\frac{1}{2} \phi^{2}\left(v_{2}^{2}+e^{2 x_{2}} v_{3}^{2}\right) .
$$

which is functionally dependent on $\left\{E, F_{1}, F_{2}, F_{3}\right\}$.

Example 3. Consider the regular Lagrangian

$$
L=\frac{1}{2} v_{1}^{2}+\frac{1}{2} x_{1}^{2}\left(v_{2}^{2}+v_{3}^{2}\right)+\alpha x_{2} v_{3},
$$

where $\alpha \in \mathbb{R}$ and $\alpha x_{1} \neq 0$. The Lagrangian (3.7) is of mechanical type, where the kinetic part is defined by the metric $g=d x_{1}^{2}+x_{1}^{2}\left(d x_{2}^{2}+d x_{3}^{2}\right)$ (see Category C metrics, in the English translation of [3], which correspond to the case of an intransitive group $G_{3}$ ). A straightforward computation shows that

$$
\begin{aligned}
\bar{D}_{t}= & \partial_{t}+v_{1} \partial_{x_{1}}+v_{2} \partial_{x_{2}}+v_{3} \partial_{x_{3}}+x_{1}\left(v_{2}^{2}+v_{3}^{2}\right) \partial_{v_{1}}+\left(\frac{\alpha v_{3}-2 x_{1} v_{1} v_{2}}{x_{1}^{2}}\right) \partial_{v_{2}} \\
& -\left(\frac{\alpha v_{2}+2 x_{1} v_{1} v_{3}}{x_{1}^{2}}\right) \partial_{v_{3}} .
\end{aligned}
$$

In this case, three simple variational symmetries are $X_{0}:=\partial_{t}, X_{1}:=\partial_{x_{2}}$ and $X_{2}:=\partial_{x_{3}}$, with associated first integrals $F_{0}:=v_{1}^{2}+x_{1}^{2}\left(v_{2}^{2}+v_{3}^{2}\right), F_{1}:=\alpha x_{3}-x_{1}^{2} v_{2}, F_{2}:=\alpha x_{2}+x_{1}^{2} v_{3}$. As $X_{1}$ and $X_{2}$ are not tangent to the 4-dimensional level manifolds $\left\{F_{i}=c_{i}, i=0,1,2\right\}$, they cannot be used to completely integrate the restriction of $\bar{D}_{t}$ to these manifolds. However, it is easy to determine the variational symmetry

$$
Y_{1}=x_{1}^{2}\left(v_{2} \partial_{x_{2}}+v_{3} \partial_{x_{3}}\right)+\alpha\left(v_{3} \partial_{v_{2}}-v_{2} \partial_{v_{3}}\right),
$$

which is tangent to $\left\{F_{i}=c_{i}, i=0,1,2\right\}$ and is linearly independent from $\bar{D}_{t}, X_{0}, X_{1}$ and $X_{2}$. We denote by $F_{3}$ the first integral associated with $Y_{1}$, and since

$$
L_{Y_{1}}(\Theta)=d\left(\frac{1}{2}\left(v_{2}^{2}+v_{3}^{2}\right) x_{1}^{4}+\alpha x_{1}^{2} x_{2} v_{3}\right)
$$

it can be readily seen that $F_{3}=x_{1}^{4}\left(v_{2}^{2}+v_{3}^{2}\right)$. Now, since $F_{0}, F_{1}, F_{2}$ and $F_{3}$ are functionally independent, one can consider the 3-dimensional level manifolds $\Upsilon_{c}:=\left\{F_{i}=c_{i}, i=0,1,2,3\right\}$ and use the adapted solvable structure $\left\{Y_{0}:=X_{0}, Y_{1}\right\}$ to completely integrate the restriction of $\mathscr{D}$ to $\Upsilon_{c}$. To this 
end, let

$$
\Upsilon_{c}=\left\{\begin{array}{l}
v_{1}=\varepsilon_{1} \frac{\sqrt{c_{0} x_{1}^{2}-c_{3}}}{x_{1}}, \quad \varepsilon_{1}= \pm 1 \\
v_{2}=\varepsilon_{2} \frac{\sqrt{c_{3}-\left(\alpha x_{2}-c_{2}\right)^{2}}}{x_{1}^{2}}, \quad \varepsilon_{2}= \pm 1 \\
v_{3}=\frac{c_{2}-\alpha x_{2}}{x_{1}^{2}}, \\
x_{3}=\varepsilon_{2} \frac{\sqrt{c_{3}-\left(\alpha x_{2}-c_{2}\right)^{2}}+c_{1}}{\alpha},
\end{array}\right.
$$

and let us take $\left\{t, x_{1}, x_{2}\right\}$ as internal coordinates on $\Upsilon_{c}$ and $\Omega_{c}=d t \wedge d x_{1} \wedge d x_{2}$ as the corresponding volume form. Hence, in $U_{\mathbf{F}}=\{\Delta \neq 0\}$, where $\left.\left.\left.\Delta=Y_{0}\right\lrcorner Y_{1}\right\lrcorner \bar{D}_{t}\right\lrcorner \Omega_{c}=x_{1}^{2} v_{1} v_{2} \neq 0$, we have the adapted solvable structure $\left\{Y_{0}, Y_{1}\right\}$ for $\mathscr{D}$. The restricted 1-forms $\Omega_{i} \mid \Upsilon_{c}$ can be written as $\Omega_{i} \mid \Upsilon_{c}=d I_{i}$, with

$$
\begin{aligned}
& I_{0}=t-\frac{\varepsilon_{1}}{c_{0}} \sqrt{c_{0} x_{1}^{2}-c_{3}}, \\
& I_{1}=-\frac{\varepsilon_{1}}{\sqrt{c_{3}}} \arctan \left(\frac{c_{3}}{\sqrt{c_{3}\left(c_{0} x_{1}^{2}-c_{3}\right)}}\right)+\frac{\varepsilon_{2}}{\alpha} \arctan \left(\frac{\alpha x_{2}-c_{2}}{\sqrt{c_{3}-\left(\alpha x_{2}-c_{2}\right)^{2}}}\right),
\end{aligned}
$$

and the solutions to the Euler-Lagrange equations can be described implicitly as $\left\{F_{0}=c_{0}, F_{1}=c_{1}, F_{2}=c_{2}, F_{3}=c_{3}, I_{0}=h_{0}, I_{1}=h_{1}\right\}$.

As a final remark we note that also in Example 3 a different integration path could have been followed, considering the Abelian algebra generated by $X_{2}, Y_{0}$ and $Y_{1}$ which provides a solvable structure adapted to the first integrals $F_{2}, F_{0}$ and $F_{3}$. More in general, the freedom one has in the choice of the first integrals defining the foliation $\Upsilon_{c}$ of $U_{\mathbf{F}}$ seems to open a wide range of possibilities allowing, for instance, the use of solvable structures adapted to both variational and non variational first integrals. This method could be particularly useful when a lack of computable symmetries makes difficult the application of Liouville theorem.

\section{References}

[1] P. Basarab-Horwath, Integrability by quadratures for systems of involutive vector fields, Ukrain. Math. J. 43 (1991) 1236-1242.

[2] M.A. Barco and G.E. Prince, Solvable symmetry structures in differential form applications, Acta Appl. Math. 66 (2001) 89-121.

[3] L. Bianchi, Sugli spazi a tre dimensioni che ammettono un gruppo continuo di movimenti, Memorie di Matematica e di Fisica della Societa Italiana delle Scienze, Serie Terza Tomo XI (1898) 267-352 (Reprint: Gen. Rel. Grav. 33 (2001) No 12).

[4] G. W. Bluman and S. C. Anco, Symmetry and Integration Methods for Differential Equations (Springer, Berlin, 2002).

[5] G. W. Bluman and G. J. Reid, New symmetries for ordinary differential equations, IMA J. Appl. Math. 40 (1988) 87-94.

[6] D. Catalano Ferraioli, Nonlocal aspects of $\lambda$-symmetries and ODEs reduction, J. Phys. A: Math Theor 40 (2007) 5479-5489. 
[7] D. Catalano Ferraioli and P. Morando, Local and nonlocal solvable structures in the reduction of ODEs, J. Phys. A: Math Theor 42 (2009) 035210 (15pp).

[8] D. Catalano Ferraioli and P. Morando, Applications of solvable structures to the nonlocal symmetryreduction of ODEs, J. Nonlin. Math. Phys. 16 (2009) 27-42.

[9] M.E. Fels, Integrating scalar ordinary differential equations with symmetry revisited, Found. Comput. Math. 7 (2007) 417-454.

[10] G. Gaeta, Nonlinear symmetries and nonlinear equations (Kluwer, Dordrecht, 1994).

[11] G. Gaeta and P. Morando, On the geometry of lambda-symmetries and PDEs reduction, J. Phys. A: Math. Gen.37 (2004) 6955-6975.

[12] M.L. Gandarias, E. Medina and C. Muriel, New symmetry reductions for some ordinary differential equations, J. Nonlin. Math. Phys. 9 (2002) 47-58.

[13] T. Hartl and C. Athorne, Solvable structures and hidden symmetries, J. Phys. A: Math Gen 27 (1994) 3463-3471

[14] O. Krupkova, The geometry of Ordinary Variational Equations, Lect. Notes Math. 1678 (Springer, Berlin, 1997).

[15] C. Muriel and J.L. Romero, New method of reduction for ordinary differential equations, IMA J. Appl. Math. 66 (2001) 111-125.

[16] C. Muriel, J.L. Romero and P.J. Olver, Variational $C^{\infty}$-symmetries and Euler-Lagrange equations, $J$. Diff. Eq. 222 (2006) 164-184.

[17] P.J. Olver Application of Lie groups to differential equations (Springer, Berlin, 1993).

[18] E. Pucci and G. Saccomandi, On the reduction methods for ordinary differential equations, J. Phys. A: Math. Gen. 35 (2002) 6145-6155.

[19] J. Sherring and G. Prince, Geometric aspects of reduction of order, Trans. Amer. Math. Soc. 334 (1992) 433-453.

[20] A. M. Vinogradov et al. Symmetries and conservation laws for differential equations of mathematical physics (American Mathematical Society, Providence, 1999). 